\title{
On Mesoscale Strain Fluctuations in Tensile Tests on Additively Manufactured 17-4PH Stainless Steel
}

\author{
Mohamed Ali BOUAZIZ1 ${ }^{1}$, Joseph MARAE DJOUDA ${ }^{1-2}$, François HILD ${ }^{2}$
}

\author{
${ }^{1}$ ERMESS, EPF-Engineering School, 3 bis rue Lakanal, 92330 Sceaux, France \\ ${ }^{2}$ Université Paris-Saclay, ENS Paris-Saclay, CNRS, LMT-Laboratoire de Mécanique et \\ Technologie, 4 avenue des Sciences, 91190 Gif-sur-Yvette, France \\ * Corresponding author. E-mail address: mohamedali.bouaziz@gmail.com
}

\begin{abstract}
Additively manufactured materials usually exhibit mesoscale heterogeneities. Mesoscale fluctuations of strain fields in notched samples made of 17-4PH Stainless steel and loaded in tension are investigated. Regularized digital image correlation enables for the analysis of strain fluctuations at different length scales. Five tests on specimen fabricated with different printing parameters are studied. It is shown that the strain fluctuations have no characteristic length scale and are essentially independent of the probed processing parameters.
\end{abstract}

Keywords: Autocorrelation, Bound Powder Extrusion, Digital image correlation; Mechanical regularization, Strain field

\section{Introduction}

Additive manufacturing ( $\mathrm{AM}$ ) or 3D printing has revolutionized the modern metal processing industry [1]. This technology allows for the fabrication of complex geometries (e.g., internal, interlocking or lattice structures), to improve the functionality of components (i.e., weight, ergonomics) or to consolidate larger systems by limiting the number of components. Powder 
Bed Fusion (PBF) and Bound Powder Extrusion (BPE) are widely used metal AM techniques. These techniques were originally developed for fabricating polymeric parts, then they were adapted for direct and indirect production of metallic parts [2].

In $\mathrm{PBF}$, the requirements in terms of process handling makes access to this technique reserved for qualified operators [3] in contrast to BPE, which shows great promise as a costeffective alternative and easy to handle process. In BPE, the building material is supplied in spooled filaments. The filament is fed into a heating unit with a nozzle in a manner very similar to standard Fused Filament Fabrication (FFF). The material is extruded through a nozzle on a platform that moves up and down in the vertical ( $\mathrm{Z}$ )-direction while the extrusion head moves in the XY plane. The "green" part contains metal powder evenly distributed in waxy polymer. After printing, BPE is followed by two post-processing steps. First, most of the polymer is dissolved in a "washing" machine. Second, the part is sintered in an oven. During the sintering process, the part shrinks to fill in the spaces opened up by the dissolved binder, yielding a dense metallic part. In contrast to beam-based additive processes, the microstructure of the part is not generated layer-wise by melting and solidifying small areas, but in a steady manner during sintering from the outside of the part to the inside in order to obtain dense parts. This feature generates different microstructures and mechanical properties [4]-[6].

Microstructure and mechanical properties of various steels produced by AM such as austenitic, duplex, martensitic and precipitation-hardened steels were widely investigated [1], [7]. Such steels exhibit different microstructures and precipitation phases, which lead to variable mechanical properties [8]-[10]. Microstructures of AM steels have fine and crystallographical textures due to rapid solidification [10]. One of the main objectives of research works on AM processed steels is to establish relationships between microstructures and observed mechanical properties, but the large number of parameters involved in such processes make it challenging. It was reported that building strategies in AM may yield oriented microstructures (e.g., for SLM, elongated columnar grains [26]). The particular grain size, texture and morphology may lead to microstructural strain heterogeneities as is the case of polycrystalline materials [11]. Since these heterogeneities affect the mechanical behavior, their understanding becomes essential.

Digital Image Correlation (DIC) is used to investigate kinematic fields of materials during loading. This technique offers an accurate way of describing deformation processes. DIC allows for multiscale measurements of displacement and strain fields [12], [13]. It was used to study AM metallic materials mainly at the macroscopic scale [10], [14]-[16]. Recently, Balit et al. [11] and Margerit et al [17] used DIC for a microstructural analysis of deformation 
patterns of additively manufactured 316L. The authors showed that an optimization of the process parameters enabled the microstructure to be controlled, and consequently the macroscopic mechanical behavior.

All the studies cited above were devoted to the characterization of PBF materials. To the authors' knowledge, no mesostructural analysis was conducted on metallic materials manufactured via Bound Powder Extrusion (BPE). The present work focuses on mesoscale strain characterization of 17-4PH stainless steel obtained via BPE. Regularized DIC is used to assess strain heterogeneities at different length scales. An experimental investigation was performed to study the behavior of $17-4 \mathrm{PH}$ stainless steel obtained by the Metal-X Markeforged process, which is based on BPE. Single edge notched tension (SENT) specimens were fabricated with two different layer thicknesses. The mesoscale experiments coupled with DIC were then carried out to obtain strain fields in uniaxial tensile tests at room temperature. Strain field heterogeneities were investigated by analyzing characteristic sizes of strain concentration zones at different length scales.

The paper is organized as follows. It starts with the presentation of the specimens, the experimental set-up and protocol. Then, regularized DIC is introduced and illustrated with a synthetic test case dealing with sinusoidal displacements. Last, five tensile tests on samples made with different processing parameters are analyzed and the relationship between characteristic sizes of strain fluctuations and processing parameters are discussed.

\section{Experimental set-up and protocol}

SENT specimens were entirely made by additive manufacturing including the notch and no subsequent machining was performed on these samples. The sample dimensions are shown in Figure 1(a). The notch size meets the recommendations of ASTM E1820 standard [18], namely, specimen width and initial 'crack' length. The specimens were printed by adding molten layers of 17-4PH filament using a Markforged 3D-printer [19]. The raw material was a polymer resin based filament loaded with metal powder. The printer unwound the material spool, heated it up to the resin melting temperature with an extrusion head, and then deposited it as successive layers according to the 3D CAD model of the sample (Figure 1(b-c)). This manufacturing process allows a limited number of parameters to be varied, which are the type of filling, the layer thickness, the number of contours and the raft (present or not). For this study, five samples were fabricated and tested (Table 1). All of them were printed as a solid parts, in a flat $\pm 45^{\circ}$ orientation. For the first three samples, the wall contour number was set to 
four, and for the other two this number was reduced to two. Two post-sintered layer heights were investigated (i.e., $125 \mu \mathrm{m}$ and $50 \mu \mathrm{m}$ ). Table 1 summarizes the printing parameters for each tested specimen. Samples 125-1 and 125-2 are repeats, as well as samples 50-2 and 50-3. Sample 50-1 was not duplicated. The printed green parts were debound and sintered in a twostep oven process.

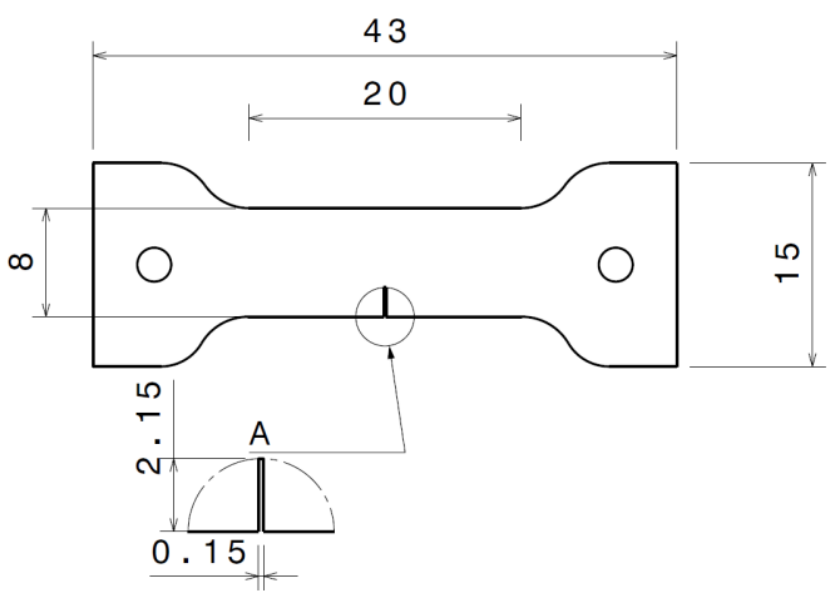

(a)

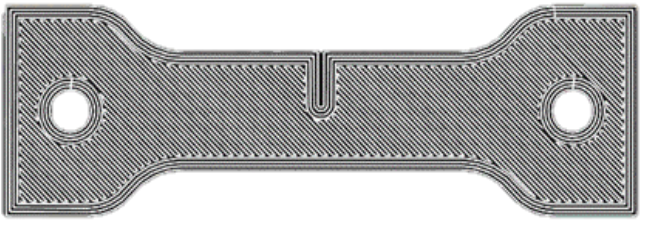

(b)

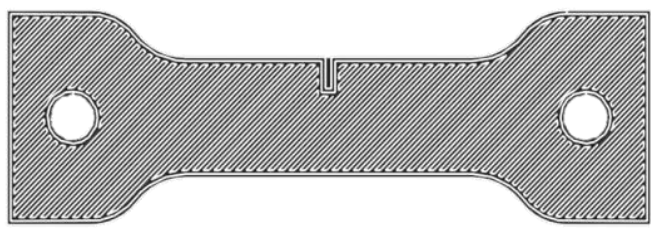

(c)

Fig. 1. (a) Sample dimensions in $\mathrm{mm}$ (thickness $=4 \mathrm{~mm}$ ). (b) One-layer nozzle path when printing samples 125-1, 125-2 and 50-1. (c) Corresponding path for samples 50-2 and 50-3

Table 1. Printing parameters and specimen designation

\begin{tabular}{|c|c|c|c|}
\hline layer thickness $(\boldsymbol{\mu m})$ & wall contour number & notch depth $(\mathbf{m m})$ & specimen designation \\
\hline \multirow{2}{*}{$\mathbf{1 2 5}$} & 4 & 3.6 & $125-1$ \\
\hline \multirow{2}{*}{$\mathbf{5 0}$} & 4 & 3.6 & $125-2$ \\
\hline & 4 & 3.6 & $50-1$ \\
\hline & 2 & 2.15 & $50-2$ \\
\hline
\end{tabular}

To enable for surface functionalization, the specimen was "mirror" polished. Grit papers of grades 400 / 600 / 1200 / 2400 / 4000 were used successively, and the polishing time with each paper was about two minutes. Further, cloths with diamond suspensions of $3 \mu \mathrm{m}$ and $1 \mu \mathrm{m}$ were also utilized. The thickness was reduced from $4 \mathrm{~mm}$ to $1.6 \mathrm{~mm}$. White speckles with micrometric dimensions were deposited using an airbrush. An exemple of speckle pattern is shown in Figure 2(a). 


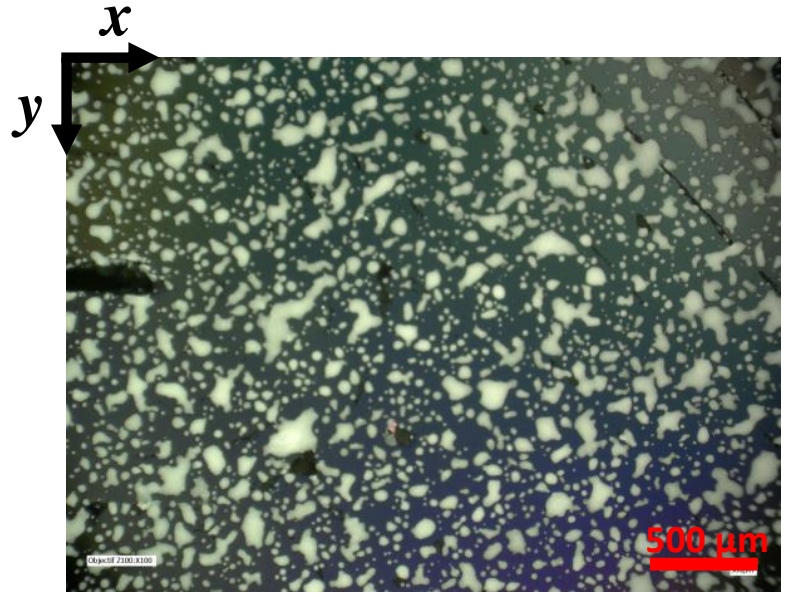

(a)

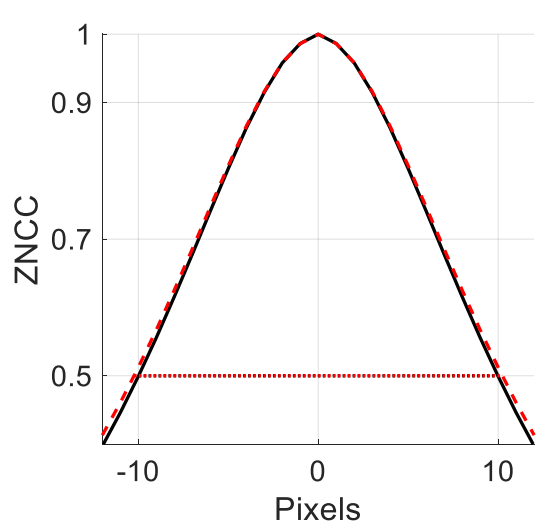

(b)

Fig. 2. (a) Speckle pattern of specimen 125-1 surface. (b) Corresponding normalized autocorrelation

The autocorrelation is used to evaluate characteristic lengths in pictures [20]. The vicinity of peaks of autocorrelations involve the shape and arrangement of texture primitives [21]. The full width at half maximum of the autocorrelation is often used to estimate characterisitic sizes of speckle patterns [22]. Figure 2(b) shows the autocorrelation of the speckle pattern displayed in Figure 2(a). The average speckle diameter is about 20 px, or equivalently $42 \mu \mathrm{m}$ (the image resolution was $2.1 \mu \mathrm{m} / \mathrm{px}$ ). It is worth noting that the two sections along the $x$ - and $y$-directions of the autocorrelation virtually coincide, which indicates that the speckles are isotropic. The average speckle diameters were assessed for all tested samples and the results are reported in Table 2. The speckle size varied between $25 \mu \mathrm{m}$ and $45 \mu \mathrm{m}$, and was essentially isotropic. No correlation was observed between layer thickness and speckle diameters.

Table 2. Average speckle diameters in $x$ and $y$ directions for the five samples

\begin{tabular}{|c|c|c|}
\hline specimen designation & $\boldsymbol{d}_{\boldsymbol{x}}(\boldsymbol{\mu \mathbf { m }})$ & $\boldsymbol{d}_{\boldsymbol{y}}(\boldsymbol{\mu} \mathbf{m})$ \\
\hline $\mathbf{1 2 5 - 1}$ & 44 & 45 \\
\hline $\mathbf{1 2 5 - 2}$ & 27 & 26 \\
\hline $\mathbf{5 0 - 1}$ & 53 & 53 \\
\hline $\mathbf{5 0 - 2}$ & 36 & 36 \\
\hline $\mathbf{5 0 - 3}$ & 41 & 42 \\
\hline Mean & 40 & 40 \\
\hline
\end{tabular}


The experimental setup was composed of a numerical Keyence VHX-1000 microscope for surface observation, a tensile micromachine and a triggering system (Table 3 ). The latter allowed images to be recorded at specific rates when the specimen was continuously loaded and each image to be related to the corresponding applied load. The crosshead velocity varied between $0.5 \mu \mathrm{m} / \mathrm{s}$ and $1.9 \mu \mathrm{m} / \mathrm{s}$ so that the global strain strain rate (i.e., related to the gauge length of the sample) ranged from $2.6 \times 10^{-5}$ and $10^{-4} \mathrm{~s}^{-1}$.

Table 3. Experimental setup parameters

\begin{tabular}{|c|c|c|c|c|c|}
\hline Microscope & \multicolumn{5}{|c|}{ Keyence VHX-1000 } \\
\hline Definition & \multicolumn{5}{|c|}{54 Megapixel 3CCD } \\
\hline Lens & \multicolumn{5}{|c|}{ VH-Z100R $(100 \times$ to $1000 \times)$} \\
\hline Image acquisition rate & \multicolumn{5}{|c|}{$1 \mathrm{fps}$} \\
\hline Load cell & \multicolumn{5}{|c|}{$5 \mathrm{kN}$} \\
\hline \multirow{2}{*}{ Crosshead velocity $(\mu \mathrm{m} / \mathrm{s})$} & 125-1 & 125-2 & $50-1$ & $50-2$ & $50-3$ \\
\hline & 1.9 & 1.1 & 1.8 & 0.5 & 0.5 \\
\hline Field of view & \multicolumn{5}{|c|}{$3.05 \times 2.28 \mathrm{~mm}^{2}$} \\
\hline Image scale & \multicolumn{5}{|c|}{$2.1 \mu \mathrm{m} / \mathrm{px}$} \\
\hline Dynamic range & \multicolumn{5}{|c|}{8 bits } \\
\hline
\end{tabular}

\section{Regularized digital image correlation}

DIC was used to measure displacement fields in all the experiments reported herein. From these raw data, Green Lagrange strain fields were assessed by exact derivation of the shape functions of the mesh made of 3-noded triangular (T3) elements.

\subsection{Digital Image Correlation principle}

DIC consists in registering two images, namely, image $f$ in the reference configuration, and image $g$ in the deformed configuration assuming gray level conservation at any pixel, $\mathbf{x}$

$$
f(\mathbf{x})=g(\mathbf{x}+\mathbf{u}(\mathbf{x}))
$$

where $\mathbf{u}$ is the unknown displacement vector. The correlation procedure aims at minimizing the sum of squared differences over the domain $\Omega$

$$
\Phi_{o}^{2}=\sum_{\Omega}[f(\mathbf{x})-g(\mathbf{x}+\mathbf{u}(\mathbf{x}))]^{2}
$$

Two different approaches of DIC are found in the literature, namely, local and global registrations. The main differences between both approaches is the registration domain $\Omega$. In 
local approches [23], small and independent domains (or subsets) are selected over the region of interest (ROI). Then, independent registrations for each of them are performed by minimizing $\Phi_{0}^{2}$. The only information kept is the displacement of the center of each interrogation window. With global approches such as finite element (FE) DIC [24], [25], which is considered herein, the domain $\Omega$ is the whole ROI. The unknowns become the nodal displacements to be determind by minimizing $\Phi_{0}^{2}$. Due to the continuity of measured displacement fields, more complex kinematics may be accessible thanks to lower uncertainties [26].

\subsection{Regularized DIC}

Regularization consists in adding some mechanical requirements with additional cost functions, for instance based on the equilibrium gap method [27]. In infinitesimal elasticity, the equilibrium gap functional reads

$$
\Phi_{m}^{2}=\{\mathbf{u}\}^{t} \cdot[\mathbf{K}]^{t} \cdot[\mathbf{K}] \cdot\{\mathbf{u}\}
$$

where $[\mathbf{K}]$ is the rectangular stiffness matrix associated with innner nodes, $\{\mathbf{u}\}$ the column vector gathering all nodal displacements. For Dirichlet edge nodes, another operator [L] is considered [28], [29]. When $\Phi_{m}^{2}$ vanishes, equilibrium is strictly satisfied for inner nodes so that mechanical admissibility is enforced.

In the following analyses, the behavior of the studied material was not necessarilly elastic. Consequently, the previous regularization may turn out to be too strong. It is thus proposed to assume Hencky-type elasticity [30], which then consists in only penalizing displacement increments $\{\delta \mathbf{u}\}$ instead of total displacements $\{\mathbf{u}\}$

$$
\Phi_{m}^{2}=\{\delta \mathbf{u}\}^{t} \cdot[\mathbf{K}]^{t} \cdot[\mathbf{K}] \cdot\{\delta \mathbf{u}\}
$$

so that the total cost function to minimize becomes

$$
\left(1+W_{m}\right) \Phi_{t}^{2}=\Phi_{o}^{2}+W_{m} \Phi_{m}^{2}
$$

where $W_{m}$ denotes the weight put on the penalty term $\Phi_{m}^{2}$. It is worth noting that the dimensions of $\Phi_{o}^{2}$ and $\Phi_{m}^{2}$ are not identical [31]. Therefore, the two cost functions are normalized with respect to their level for a trial displacement field. A plane wave of unity amplitude is considered 


$$
\mathbf{v}=\sin \left(k_{m} \xi\right) \mathbf{e}
$$

along the direction $\mathbf{e}$ of the largest edge of the circumscribed rectangle to the considered ROI,

with $\xi$ the corresponding coordinate, and $k_{m}$ the considered wavenumber. The normalized cost functions read

$$
\widetilde{\Phi}_{m}^{2}=\frac{\Phi_{m}^{2}}{\{\mathbf{v}\}^{t} \cdot[\mathbf{K}]^{t} \cdot[\mathbf{K}] \cdot\{\mathbf{v}\}} \quad \text { and } \quad \widetilde{\Phi}_{o}^{2}=\frac{\Phi_{o}^{2}}{\{\mathbf{v}\}^{t} \cdot[\mathbf{M}] \cdot\{\mathbf{v}\}}
$$

where $[\mathbf{M}]$ is the DIC Hessian matrix. The global minimization is performed on the weighted sum of the two normalized cost functions [38]

$$
\left(1+w_{m}\right) \Phi_{t}^{2}=\tilde{\Phi}_{o}^{2}+w_{m} \tilde{\Phi}_{m}^{2}
$$

where the weight $w_{m}$ put on functional $\Phi_{m}^{2}$ is proportional to a regularization $\ell_{m}$ raised to the power 4

$$
w_{m}=\left(k_{m} \ell_{m}\right)^{4}
$$

In practice, $k=2 \pi / L_{R O I}$ where $L_{R O I}$ is half the largest edge of the circonscribed rectangle to the ROI.

\subsection{Artificial test case}

To illustrate the effect of mechanical regularization, sinusoidal displacements in both directions were prescribed to a reference image for eleven different wavenumbers $k_{x}=k_{y}=$ $k$ with an amplitude $v_{0 x}=v_{0 y}=1 \mathrm{px}$. Special care was taken to perform such operation. A 'coordinate pullback' procedure was followed. It consists in determining the position in the reference configuration such that its counterpart in the deformed configuration be integervalued. Once the position in the reference configuration is known, a gray level interpolation scheme is used (here via splines) to determine the corresponding gray level. One advantage of such procedure is that gridded interpolants can be used with higher order interpolation schemes in comparison with scattered interpolants had the pullback procedure not been used.

With these deformed pictures, regularized DIC was run to measure displacements with different regularization lengths as used throughout the paper (i.e., $\ell_{m}=10,20,30,40 \mathrm{px}$ ). To analyze the influence of the latter, a fine structured mesh was created with T3 elements. The size of each element, $\ell_{F E}$, which is defined as the square root of its surface, was equal to $5 \mathrm{px}$ 
so that the reported results were controlled by the regularization length and not the finite element discretization of the displacement field (i.e., $\ell_{m}>\ell_{F E}$ ). For each analyzed case, the actual displacement amplitude $u_{m}$ was determined by interpolating the measured displacement field with a sinewave of identical wavenumber as for the prescribed displacement fields. From this result, the corresponding gain $u_{m} / u_{0}$ was obtained and is reported in Figure 3(a) where the dimensionless wavenumber $\kappa$ is defined as

$$
\kappa=\frac{k L_{0}}{2 \pi}
$$

and $L_{0}$ is the image size (here equal to $527 \mathrm{px}$ ). As expected from the previous discussions, it is observed that the higher the regularization length, the sooner the gain decays in comparison to lower regularization lengths. Further, the asymptotic response is that of a fourth-order filter (i.e. $80 \mathrm{~dB}$ per decade fall off) as anticipated from the power four dependence of the regularization weight on the regularization length (see Equation (9)). This plot thus illustrates the 'frequency response function' of regularized DIC. For low wavenumbers, the measurements are not filtered out and DIC is measuring the corresponding amplitudes (as if unregularised). Conversely, for high wavenumbers, the displacement fluctuations that are not mechanically admissible are filtered out. This response corresponds to that of a low-pass filter whose spatial cut-off frequency is controlled by the regularization length $\ell_{m}$ (Figure 3(a)).

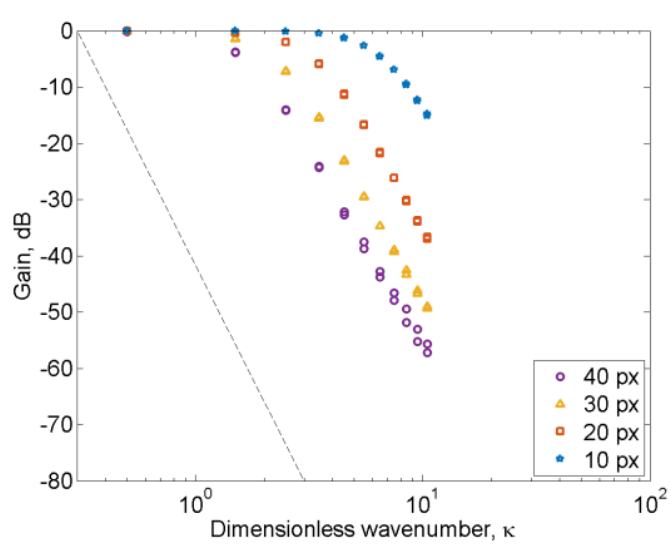

(a)

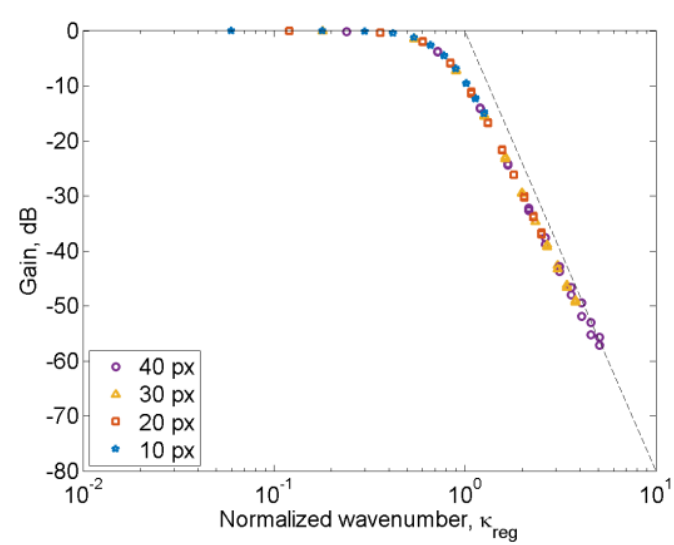

(b)

Fig. 3. (a) Decibel-gain as a function of dimensionless wavenumber $\kappa$ for the four regularization lengths $\ell_{m}$ of DIC analyses. (b) Corresponding decibel-gain vs. normalized wavenumber $\kappa_{\text {reg. }}$. The black dashed lines depict $80 \mathrm{~dB}$ per decade fall off 
All these results can be collapsed onto a single master plot (Figure 3(b)) that defines the transfer function of the considered filter, namely, the decibel gain as a function of the normalized wavenumber $\kappa_{\text {reg }}$

$$
\kappa_{\text {reg }}=\kappa \frac{2 \pi \ell_{m}}{L_{\mathrm{ROI}}}
$$

where $L_{\mathrm{ROI}}$ is the maximum size of the considered ROI. Equation (11) shows that the effective regularization length is equal to $2 \pi \ell_{m}$. In this setting, the cut-off wavenumber corresponds to $\kappa_{\text {reg }}=1$ (Figure $3(\mathrm{~b})$ ). The effect of regularization is then understood as changing the normalized cut-off wavenumber $\kappa_{c}$ of the fourth order mechanical filter

$$
\kappa_{c}=\frac{L_{\mathrm{ROI}}}{2 \pi \ell_{m}}
$$

or equivalently, the cut-off wavelength $\lambda_{c}$ reads

$$
\lambda_{c}=2 \pi \ell_{m} \frac{L_{\mathrm{ROI}}}{L_{0}}
$$

As already mentioned, the effective regularization length is equal to $2 \pi \ell_{m}$ in the present setting. Equation (13) shows that the higher the regularization length $\ell_{m}$ (i.e. the higher the weight in the penalty term), the higher the corresponding cut-off wavelength (i.e. more displacement fluctuations are filtered out when they are not mechanically admissible).

To assess the registration quality, the root mean square (RMS) gray level residuals are reported for all analyzed cases in Figure 4(a). As the regularization length increased, the residuals started to increase earlier as more weight was put on the penalty term (i.e., displacements were filtered out earlier). As a consequence, the measured displacements were no longer close to the actual (i.e., prescribed) solution. This observation shows that the residuals are very useful in detecting discrepancies between measured and actual displacements. When the regularization length is increased, so is the regularization weight, more constraints are added to DIC and the consequence is that residuals increased. 


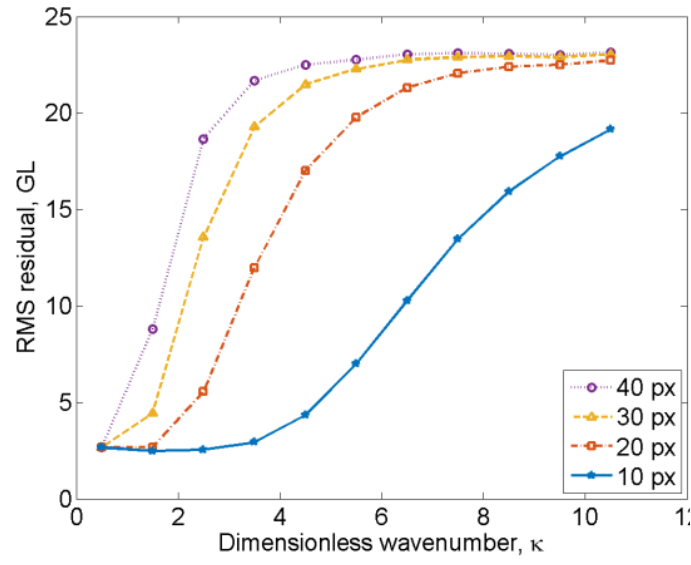

(a)

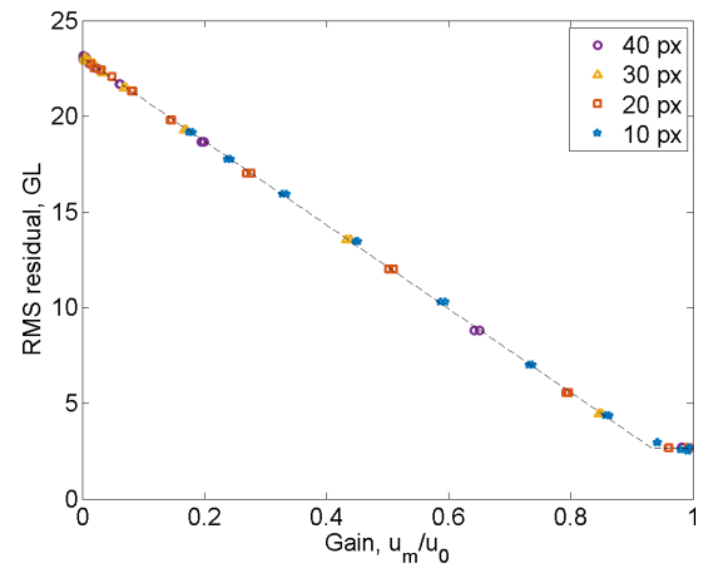

(b)

Fig. 4. (a) RMS residuals vs. dimensionless wavenumber $\kappa$ for different regularization lengths $\ell_{m}$. (b) RMS residuals vs. amplitude gain for different regularization lengths $\ell_{m}$. The black dashed line shows a piecewise linear trend

Figure 4(b) shows the dependence of the measured amplitude $u_{m}$ with the RMS residuals for the investigated regularization lengths. The lower the gain, the higher the RMS residual. It is worth noting that the RMS residual does not cancel out for gains close to 1 . This phenomenon is due to the underlying finite element discretization of the measured displacement field that does not allow the residuals to vanish for low wavenumbers. Moreover, there is a quasi-perfect collapse irrespective of the regularization length when a piecewise linear interpolation is considered. For gains greater than 0.93 , the residuals are less sensitive to deviations from 1 . Conversely, for a gain less than 0.93 , the residuals increase linearly when the gain varies. This result further emphasizes the usefulness of gray level residuals as quality indicator of DIC results. This is particularly true when the actual solution is unknown as occurs in many experiments (e.g., those reported hereafter).

\section{$4 \quad$ Results and discussions}

\subsection{Notch opening displacement}

To characterize the kinematics of notched samples, notch opening displacements (NODs) were measured via DIC by calculating jumps as the vertical mean displacement difference between two zones chosen around notches (Figure 5(a)) [32], [33]. The zone size was equal to $100 \times 100$ px $($ or $210 \times 210 \mu \mathrm{m})$ and meshed with 9 px elements. 


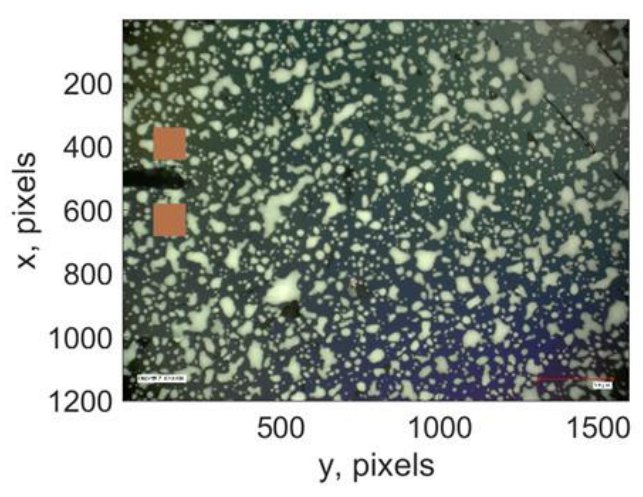

(a)

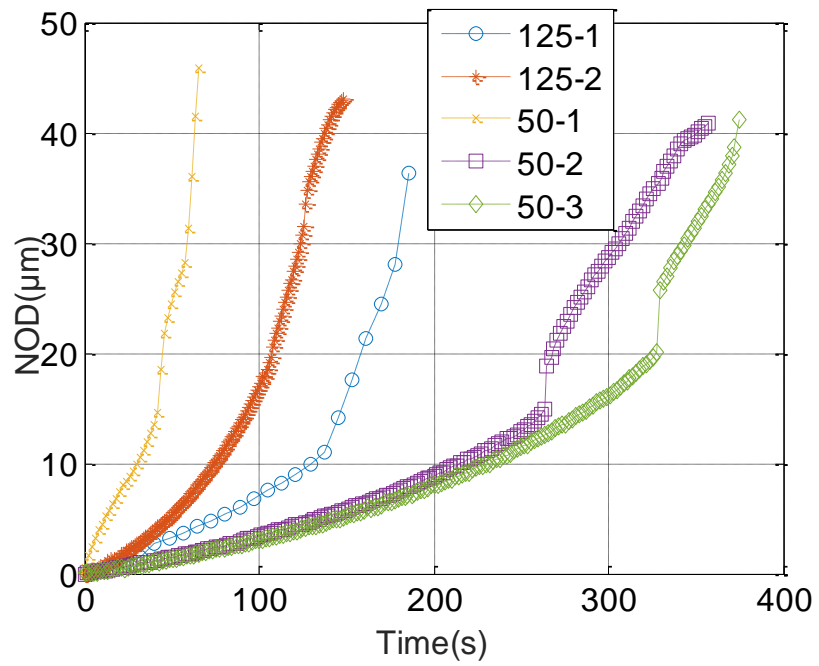

(b)

Fig. 5. (a) ROIs used to measure NODs (125-1 sample). (b) NOD histories for the five tensile tests

The analyses were performed for all images of each test. Table 4 gathers the DIC parameters used for this preliminary analysis. As only mean translations were sought to measure NODs, the regularization length was selected equal to $50 \mathrm{px}$. With such parameters, the NOD noise floor was very low for all five tests.

Table 4. DIC analysis parameters for NOD measurements

\begin{tabular}{|l|l|}
\hline DIC software & Correli $3.0[34]$ \\
\hline ROI size & $2 \times(100 \times 100)$ pixels (Fig. 5(a)) \\
\hline Element size & $\ell_{F E}=9 \mathrm{px}$ \\
\hline Mechanical regularization length & $50 \mathrm{px}$ \\
\hline Matching criterion & regularized sum of square differences \\
\hline NOD noise-floor $(\mathbf{n m})$ & 3 \\
\hline
\end{tabular}

Figure 5(b) reports the NOD variations for all tested samples. The NOD histories varied from one test to another because the crosshead velocity was not identical (Table 3). Further, failure occurred for NODs values around $40 \mu \mathrm{m}$ (Table 5). The measured NODs at failure were very close with a coefficient of variation of $8.5 \%$. This result demonstrates that, despite differences between tested samples (notch deph and layer thikness when printing), failure occurred for very close NOD levels, which means that the manufacturing process had good repeatability, in particular for the $50-\mu \mathrm{m}$ layer samples. 
Table 5. NOD at failure for the five analyzed tests

\begin{tabular}{|c|c|}
\hline Specimen designation & NOD $_{\mathbf{c}}(\boldsymbol{\mu m})$ \\
\hline $\mathbf{1 2 5}-1$ & 36 \\
\hline $\mathbf{1 2 5}-2$ & 43 \\
\hline $\mathbf{5 0 - 1}$ & 46 \\
\hline $\mathbf{5 0 - 2}$ & 41 \\
\hline $\mathbf{5 0 - 3}$ & 41 \\
\hline Mean & $\mathbf{4 1}$ \\
\hline Standard deviation & $\mathbf{3 . 5}$ \\
\hline
\end{tabular}

\subsection{Kinematic fields}

Displacements fields of ROIs ahead of the notch root were measured via DIC for all tested samples. The DIC analysis parameters are gathered in Table 6.

Table 6. DIC analysis parameters

\begin{tabular}{|l|l|}
\hline DIC software & Correli $3.0[34]$ \\
\hline ROI size & $800 \times 1000$ pixels \\
\hline Element size & $\ell_{F E}=4$ pixels \\
\hline Mechanical regularization length [28] & $10,20,30,40$ pixels \\
\hline Matching criterion & regularized sum of square differences \\
\hline Strain calculation & exact derivation of shape functions \\
\hline Strain window size & element size \\
\hline
\end{tabular}

To evaluate the measurement uncertainties, preliminary DIC analyses were conducted on 10 images prior to the tests on samples 50-2 and 50-3. The standard uncertainties were estimated for each considered regularization length by calculating the noise-foor level for longitudinal displacement and strain fields (Figure 6). For both tests, the noise floor levels decrease with $\ell_{m}$. Power laws interpolate very well the experimental observations. For displacements, a power close to -1 is observed, which indicates that the uncertainties are essentially controlled by acquisition noise. Similarly, a slope of -2 for strains is the signature of acquisition noise. These results show that measurement uncertainties can be reduced by increasing the regularization length since random fluctuations are not mechnically admissible and thus filtred out. 


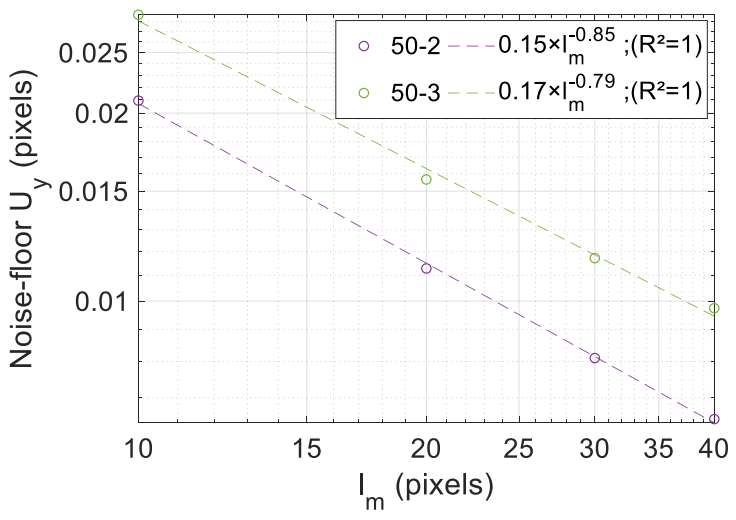

(a)

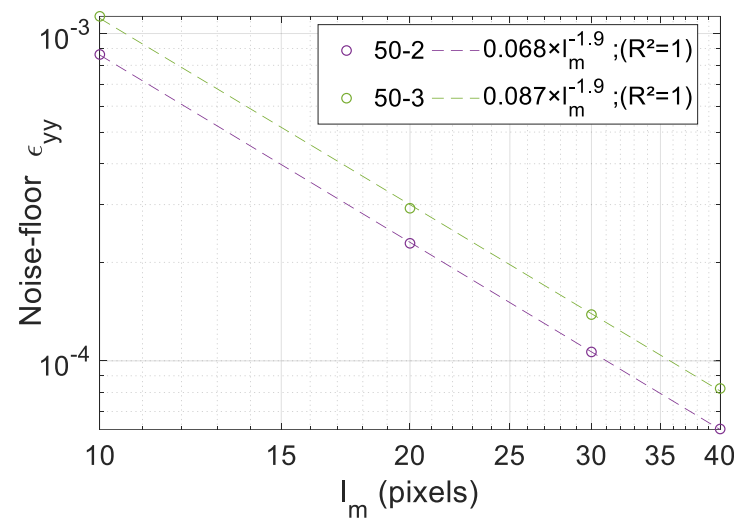

(b)

Fig. 6. Noise floor levels as functions of regularization length for longitudinal (a) displacement and (b) strain fields

In Figure 7, the longitudinal strain fields at identical NOD level $(20 \mu \mathrm{m})$ are shown for the five tests. Three aspects are noted:

- strain concentrations in the vicinity of the notch root, which was expected given the geometry of the tested samples (Figure 1(a));

- strained bands in the immediate vicinity of the notch (mainly in tests 50-2, 125-1 and 125-2), which are due to the contours generated when printing the notches (Figure 1(bc));

- Farther away from the notch root, the strain fields have a 'granular' pattern.

The first two points were expected. However, the third one was unexpected because its origin is unknown. This pattern is common to all strain fields. The aim of the following analyses is to charaterize such patterns and investigate their dependence on the printing parameters that were not identical in all reported cases (Table 1). 


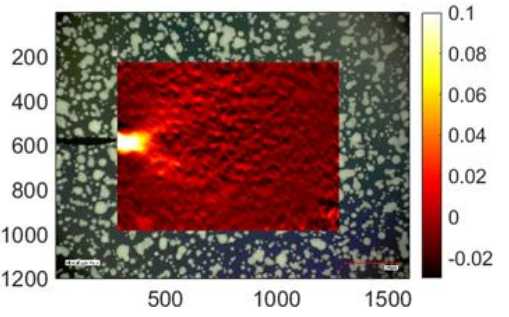

(a)

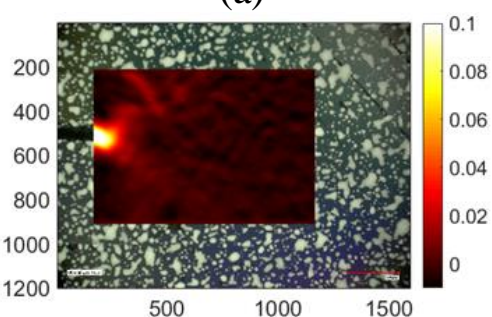

(d)

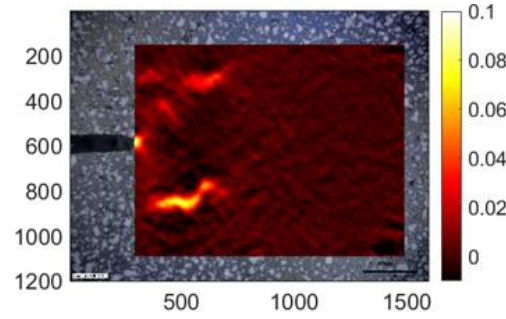

(b)

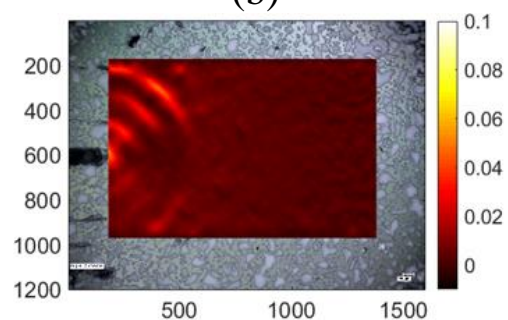

(e)

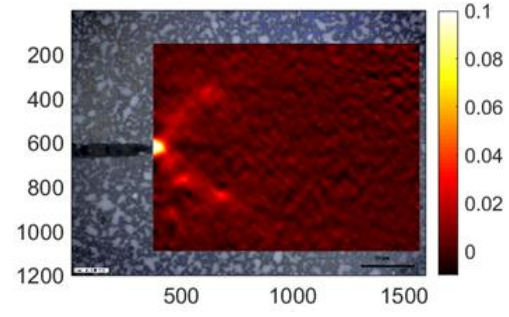

(c)

Fig. 7. Longitudinal strain fields when NOD $\approx 20 \mu \mathrm{m}$ and $\ell_{m}=30 \mathrm{px}$. (a) 50-1, (b) 50-2, (c) 50-3, (d) 125-1 and (e) 125-2 samples.

Figure 8 shows the mean longitudinal strain vs. NOD curve for specimen 50-3. The mean longitudinal strain was computed from the strains fields measured in each ROI. This plot is essentially monotonic except around 20-25 $\mu \mathrm{m}$ levels for the NOD. In that interval, crack initiation occurred, which led to NOD / strain jumps. The strain fields reported for several NODs show that the granular pattern persisted throughout the test. It also shows, from visual inspection, that the strain pattern size was virtually constant. 

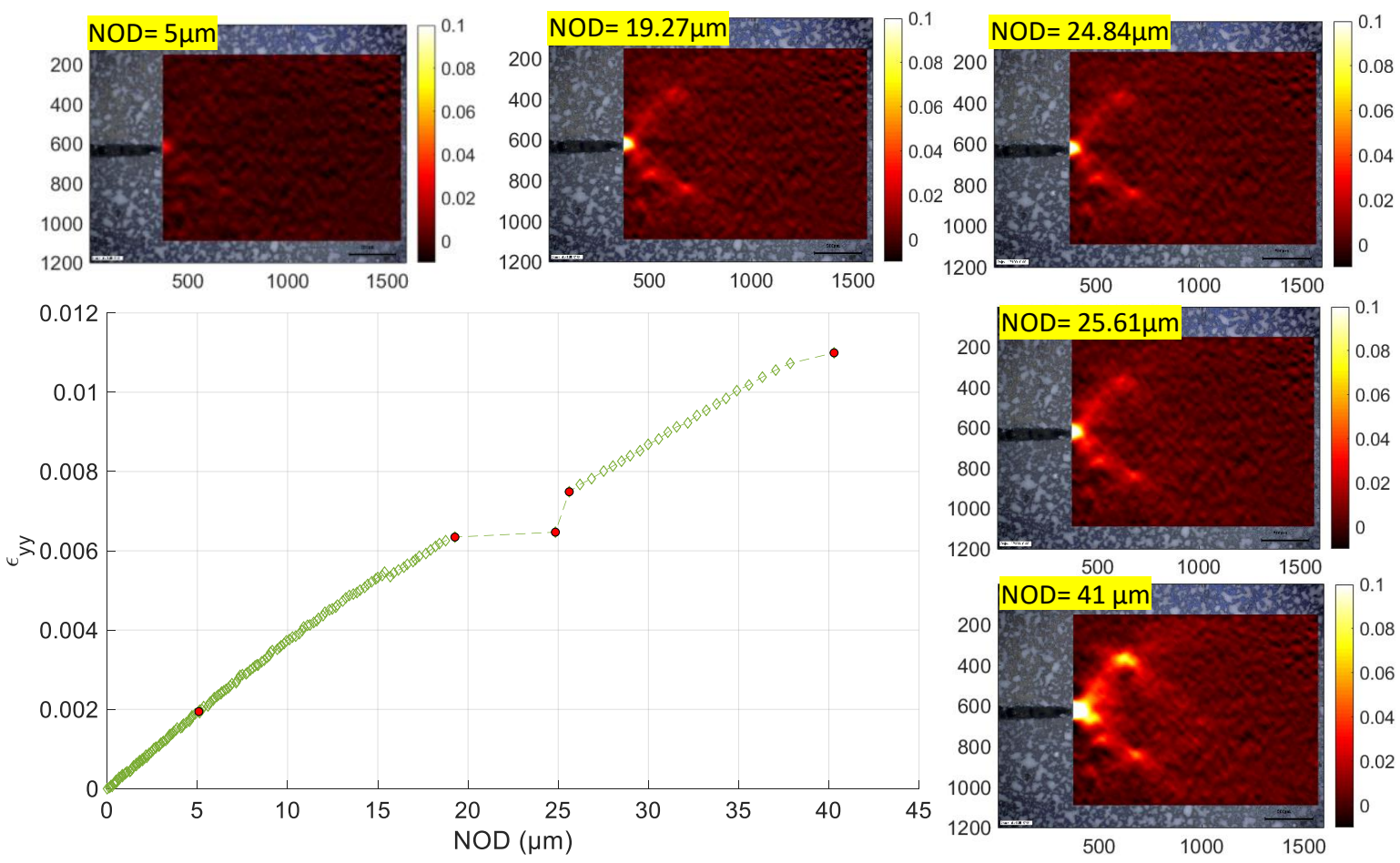

Fig. 8. Average longitudinal strain vs. NOD for sample 50-3. Strain fields are ploted for several NODs depicted by the red points

\subsection{Sizes of strain concentrations zones}

As introduced above, the full width at half maximum of the autocorrelation is often used to estimate characteristic sizes of speckle patterns [35], [24]. Here the autocorrelation was applied to strain fields [36] to estimate the length along the transverse (i.e. $d_{x}$ ) and longitudinal (i.e. $d_{y}$ ) directions of the strain pattern. Figure 9(a) shows the normalized autocorrelation of the strain field measured for the last loading step of sample 50-3 (see Figure 8 when NOD = 41 $\mu \mathrm{m}$ ). For this sample, the strain field patterns were elongated in the transverse direction. Details of the autocorrelation about its maximum (centred at 0) are shown in Figure 9(b). The full widths at half maximum were determined along the longitudinal and transverse directions. In the sequel, these quantities are referred to as pattern diameters. 


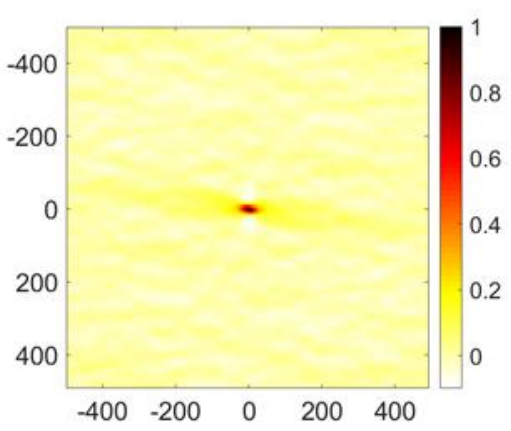

(a)

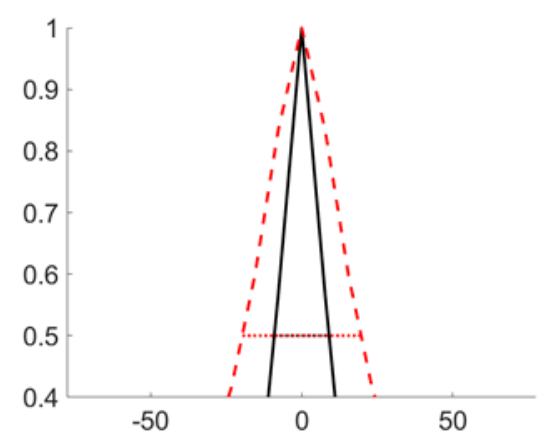

(b)

Fig. 9. (a) Normalized autocorrelation of the longitudinal strain field for the last loading step of sample 50-3 (Figure 8). (b) Corresponding profiles along both directions. Evaluation of the pattern diameters as full widths at half maximum

Figure 10 shows the pattern diameters $\left(d_{x}\right.$ and $\left.d_{y}\right)$ histories for all tested samples. The strain patterns are elongated in the longitudinal direction since $d_{y}$ is greater than $d_{x}$ for any of the considered tests. It is observed that the pattern sizes are essentially constant for all steps and for all tested samples. Further, all pattern sizes are very close except test $125-1$. The average pattern diameters in the transverse direction $d_{x}$ is equal to $\approx 50 \mu \mathrm{m}$, and $d_{y} \approx 70 \mu \mathrm{m}$ in the longitudinal direction. At the end of some tests, the pattern sizes increased. Such trend is related to strain concentrations that occur after crack initiation in the vicinity of the notch root. The dominant feature is no longer the granular pattern (Figure 7) but strained bands due to damage with larger characteristic sizes. It is worth noting that all these results were obtained for the same regularization length (i.e. $\ell_{m}=30 \mathrm{px}$ ). The effect of $\ell_{m}$ is analyzed in the sequel.

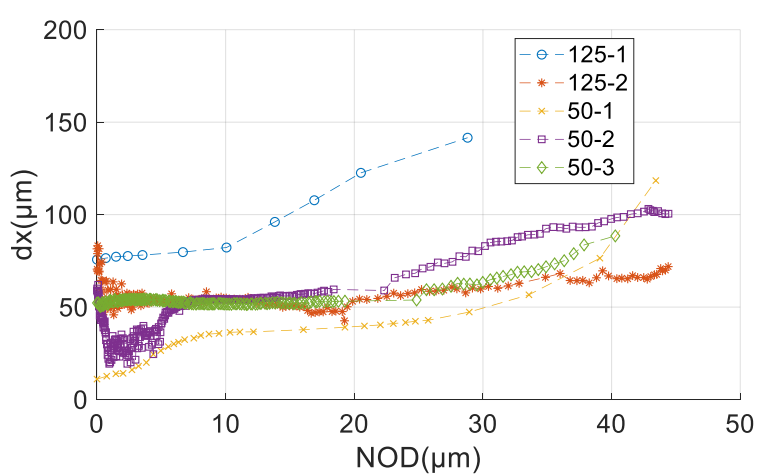

(a)

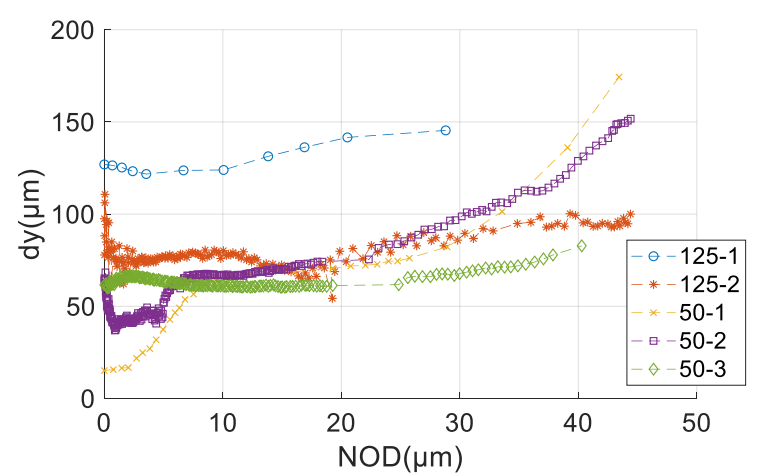

(b)

Fig. 10. Pattern diameters histories for all five tests when the regularization length was equal to $30 \mathrm{px}$ 


\subsection{Effect of regularization length}

The mechanical regularization used herein allows the cut-off wavelength to be tailored by varying the regularization length $\ell_{m}$ (Section 3 ). In the present analyses, a relaxation procedure was followed [28]. It consisted in starting the registrations with the largest regularization length (here $\ell_{m}=40 \mathrm{px}$ ) to capture large wavelength components of the measured displacement fields. Once convergence was achieved, a new analysis was started with a lower regularization and initialized with the previous results. Finer kinematic details were then captured. Following such relaxation route, four different regularization lengths were considered $\left(\ell_{m}=40,30,20\right.$ and $10 \mathrm{px})$.

The effect of selecting different regularization lengths is illustrated in Figure 11 for the longitudinal strain field of sample $50-3$ prior to crack initiation (i.e. NOD $\approx 20 \mu \mathrm{m}$ ). As the regularization length increases, the strain fluctuations decrease as a result of mechanical filtering. Visual inspection of strain fields also shows that the concentration zones spread more and more when $\ell_{m}$ increases. As a consequence, the characterisitc sizes of the deformation pattern are expected to increase with $\ell_{m}$.

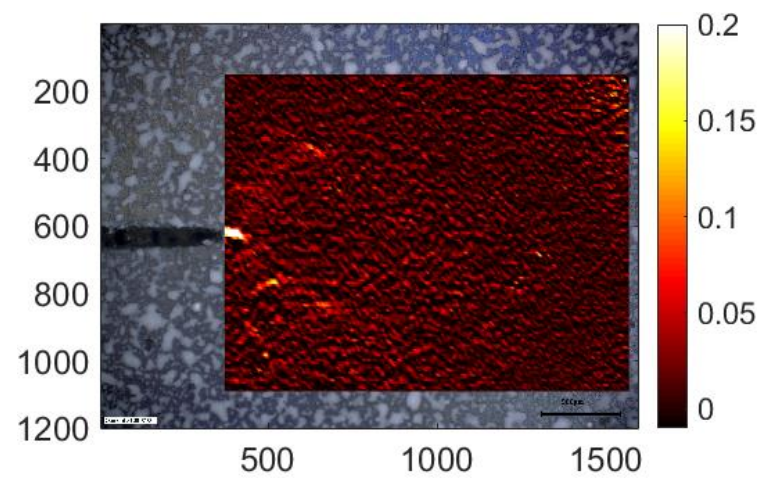

(a)

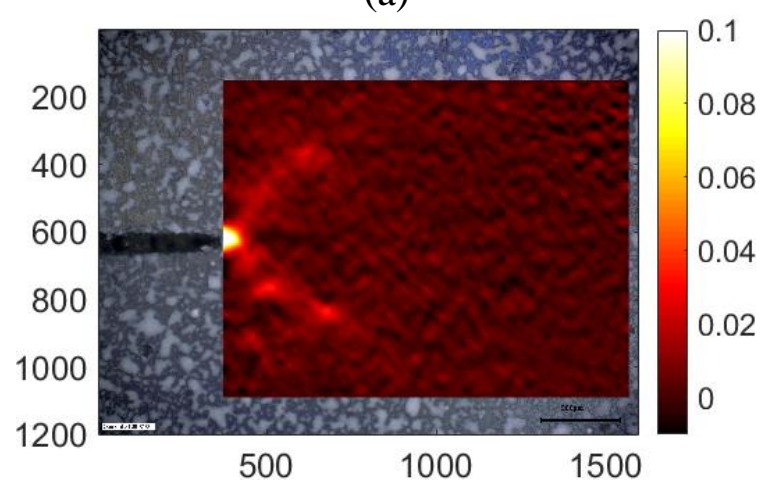

(c)

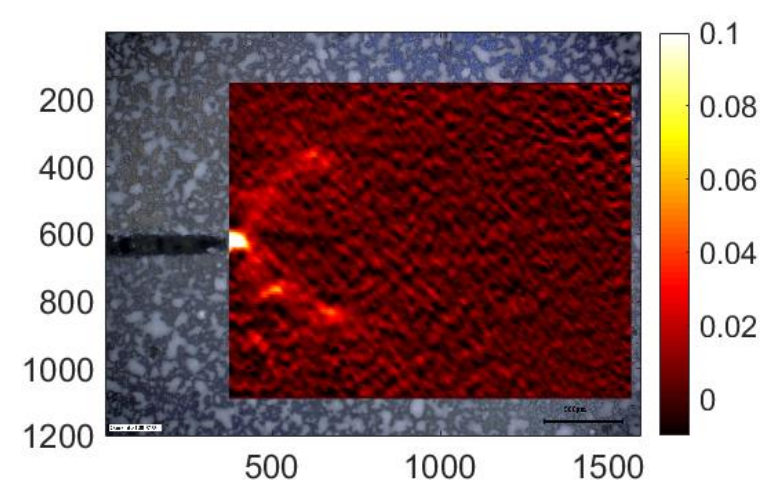

(b)

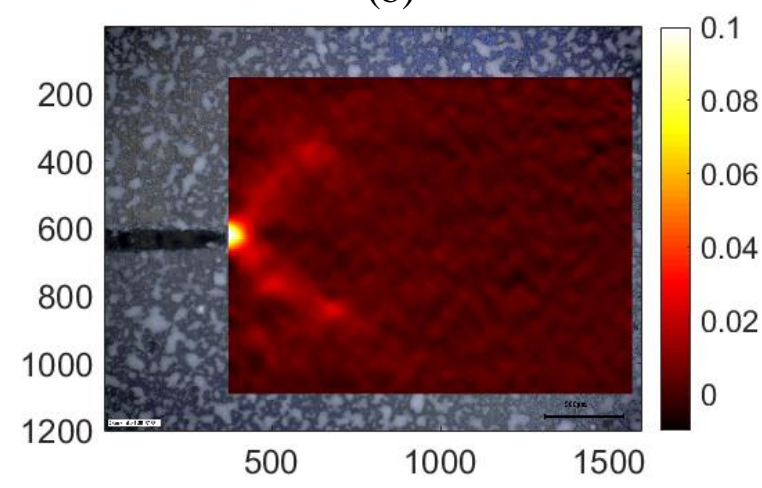

(d)

Fig. 11. Longitudinal strain field for sample 50-3 $(\mathrm{NOD}=19.3 \mu \mathrm{m})$. Four regularization lengths $\ell_{m}$ were considered: (a) 10, (b) 20, (c) 30 , and (d) 40 px 
Figure 12 shows the corresponding root mean square (RMS) gray level residuals for the four regularization lengths. Their level remains low for all considered regularisation lengths throughout the whole test. Their gradual increase after crack initiation (i.e. NOD $>20 \mu \mathrm{m}$ ) is due to the development of damaged zones in the vicinity of the notch root (Figure 8). As the regularization length is decreased, so do the residuals (i.e. the kinematics is better captured since it is no longer strongly filtered and more strain fluctuations are allowed). This trend was expected from the results reported in Section 3 (Figure 4). However, the decay remains modest given the fourfold variation in regularization length. Last, let us note that the temporal fluctuations of the gray level residuals prior to crack initiation are believed to be associated with Portevin Le Chatelier phenomena (PLC), which are known to occur in 17-4 PH steels at low strain rates or high temperatures [37] [38], [39]. In the present cases, the average longitudinal strain rate ranged from $1.2 \times 10^{-5}$ to $10^{-4} \mathrm{~s}^{-1}$ when evaluated in the analyzed ROIs (Appendix A).

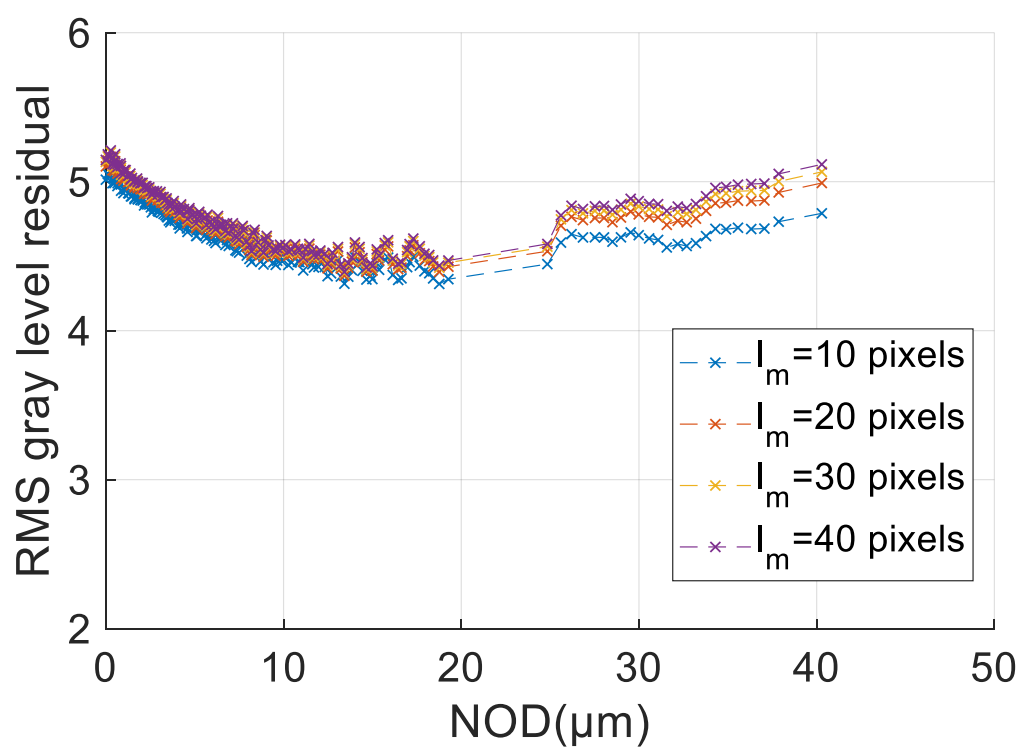

Fig. 12. RMS gray level residuals in four DIC analyses of test 50-3 with different regularization lengths $\ell_{m}$.

The dependence of characteristic sizes of strain field patterns is investigated for different regularization lengths $\ell_{m}$. For any regularization length, both characteristic sizes reported in Figure 13 are weakly dependent on the NOD level prior to crack initiation. This is no longer true after crack initiation as was already discussed above. Further, the lower the regularization length, the lower the characteristic sizes measured by $d_{x}$ and $d_{y}$. This result indicates that strain 
fluctuations occcur at different scales and there is no unique length scale concerned by these fluctuations.

From all these analyses, it is concluded that the strain fluctuations prior to crack initiation are due to Portevin Le Chatelier effects. In the present case, it occurred for mean strain rates ranging from computed in each considered ROI. For the studied test, no unique length scale was found as the characteristic pattern sizes varied with the regularization length. The following section summarizes the results for all five samples.

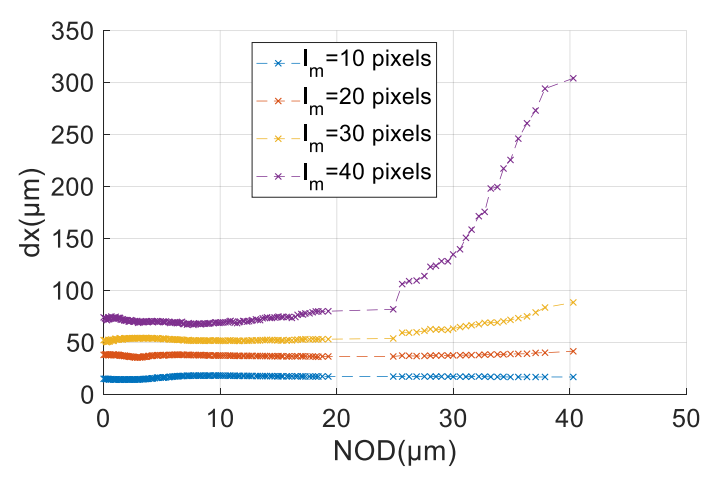

(a)

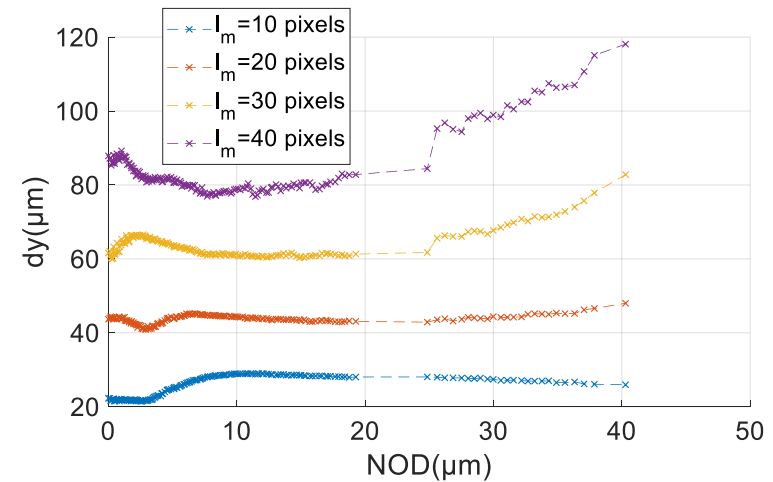

(b)

Fig. 13. Pattern diameters histories for sample 50-3 obtained with different regularization lengths $\ell_{m}$

\subsection{Mean size of strain concentration zones}

The same type of investigation was carried out for all five tests. The results are gathered in Figure 14 in which the mean area $d_{x} d_{y}$ of strained zones is plotted (in log-log scale) as function of the regularisation length $\ell_{m}$. For all tests, the mean pattern area increases with $\ell_{m}$. More precisely, power laws interpolate very well the experimental observations (i.e. with coefficients of determination very close to 1$)$. All powers are very close to $2\left(\alpha=2^{ \pm 0.2}\right)$. These last results show that there is no characteritic length scale in the longitudinal strain fields and that the scaling is that of the space dimension. 


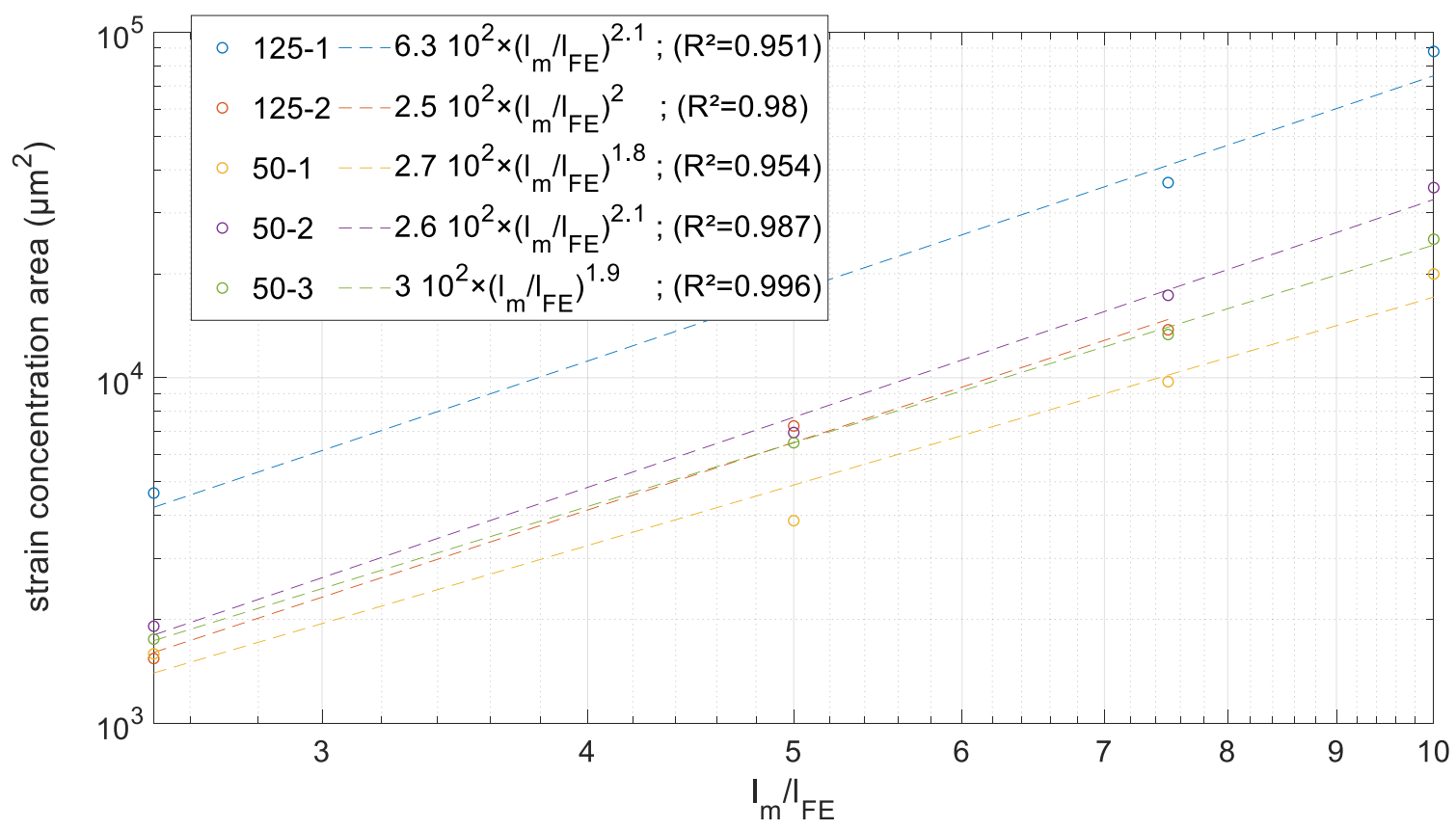

Fig. 14. Mean area of strain concentration zone as function of the regularization length $\ell_{m}$ normalized by the element size $\ell_{F E}$

Since different printing parameters were considered (Table 1), it is observed that except sample 125-1, which experienced strain fluctuations with higher characteristic sizes, all other samples had very close characteristic sizes for their longitudinal strain fluctuations. It is concluded that such phenomena are not controlled by the printing parameters investigated herein.

\section{Conclusion}

Five single edge notched samples made of additively manufactured 17-4PH steel were loaded in tension. Optical images with micrometer resolution were acquired during these tests. Digital image correlation (DIC) analyses were conducted in order to measure kinematic fields. Strain field histories highlight specific features such as strain concentrations close to the notch root, strained bands that are inherent to AM for notched specimen (contours), and 'granular' patterns far from the strain concentrations of notches and contours. The use of regularized DIC enabled different length scales to be investigated by varying the regularization length by a factor up to 4. Characteritic sizes of longitudinal strain fluctuations were assessed thanks to the autocorrelation of the measured strain fields. It is believed that the temporal fluctuations were mostly related to Portevin Le Chatelier effects. The development of motionless spatial fluctuations were shown to occur at different length scales and were essentially independent of the printing parameters investigated herein. 
These observations show that Portevin Le Chatelier effects occur in AM 17-4PH alloys at very low strain rates (i.e., $10^{-5}$ to $10^{-4} \mathrm{~s}^{-1}$ ) and at room temperature in single edge notched samples loaded in tension. Further, spatial strain fluctuations occurred with no characteristic length scale for regions of the order of $4 \mathrm{~mm}^{2}$ in surface area.

\section{References}

[1] A. Bandyopadhyay, Y. Zhang, and S. Bose, "Recent developments in metal additive manufacturing," Curr. Opin. Chem. Eng., vol. 28, pp. 96-104, 2020.

[2] J. Gonzalez-Gutierrez, S. Cano, S. Schuschnigg, C. Kukla, J. Sapkota, and C. Holzer, "Additive manufacturing of metallic and ceramic components by the material extrusion of highly-filled polymers: A review and future perspectives," Materials (Basel),, vol. 11, no. $5,2018$.

[3] R. Bibb, "Physical reproduction - rapid prototyping technologies," in Medical Modelling, Elsevier, 2006, pp. 59-96.

[4] A. Simchi, A. Rota, and P. Imgrund, "An investigation on the sintering behavior of 316L and 17-4PH stainless steel powders for graded composites," Mater. Sci. Eng. A, vol. 424, no. 1-2, pp. 282-289, May 2006.

[5] S. J. Kang, Sintering. Elsevier Ltd, 2005.

[6] Y. Thompson, J. Gonzalez-Gutierrez, C. Kukla, and P. Felfer, "Fused filament fabrication, debinding and sintering as a low cost additive manufacturing method of 316L stainless steel,” Addit. Manuf., vol. 30, p. 100861, Dec. 2019.

[7] P. Bajaj, A. Hariharan, A. Kini, P. Kürnsteiner, D. Raabe, and E. A. Jägle, "Steels in additive manufacturing: A review of their microstructure and properties," Mater. Sci. Eng. A, vol. 772, no. November 2019, 2020.

[8] A. Yadollahi, M. Mahmoudi, A. Elwany, H. Doude, L. Bian, and J. C. Newman, "Effects of crack orientation and heat treatment on fatigue-crack-growth behavior of AM 17-4 PH stainless steel,” Eng. Fract. Mech., vol. 226, no. November 2019, p. 106874, 2020.

[9] Q. Portella, M. Chemkhi, and D. Retraint, "Influence of Surface Mechanical Attrition Treatment (SMAT) post-treatment on microstructural, mechanical and tensile behaviour of additive manufactured AISI 316L," Mater. Charact., vol. 167, no. June, p. 110463 , 2020. 
[10] J. J. Yan, M. T. Chen, W. M. Quach, M. Yan, and B. Young, "Mechanical properties and cross-sectional behavior of additively manufactured high strength steel tubular sections," Thin-Walled Struct., vol. 144, no. April, p. 106158, 2019.

[11] Y. Balit, E. Charkaluk, and A. Constantinescu, "Digital image correlation for microstructural analysis of deformation pattern in additively manufactured $316 \mathrm{~L}$ thin walls," Addit. Manuf., vol. 31, no. November 2019, p. 100862, 2020.

[12] F. Hild, A. Misra, and F. Dell'Isola, "Multiscale DIC applied to Pantographic Structures," Exp Mech, vol. 61, pp. 431-443, 2020.

[13] F. Hild, B. Raka, M. Baudequin, S. Roux, and F. Cantelaube, "Multiscale displacement field measurements of compressed mineral-wool samples by digital image correlation," Appl. Opt., vol. 41, no. 32, p. 6815, 2002.

[14] D. Foehring, H. B. Chew, and J. Lambros, "Characterizing the tensile behavior of additively manufactured Ti-6Al-4V using multiscale digital image correlation," Mater. Sci. Eng. A, vol. 724, no. December 2017, pp. 536-546, 2018.

[15] M. S. Pham, B. Dovgyy, and P. A. Hooper, "Twinning induced plasticity in austenitic stainless steel 316L made by additive manufacturing," Mater. Sci. Eng. A, vol. 704, no. April, pp. 102-111, 2017.

[16] Z. Zeng et al., "Wire and arc additive manufacturing of a Ni-rich NiTi shape memory alloy: Microstructure and mechanical properties," Addit. Manuf., vol. 32, no. September 2019, 2020.

[17] P. Margerit, D. Weisz-Patrault, K. Ravi-Chandar, and A. Constantinescu, "Tensile and ductile fracture properties of as-printed 316L stainless steel thin walls obtained by directed energy deposition,” Addit. Manuf., vol. 37, p. 101664, Jan. 2021.

[18] ASTM-E1820-11, "Standard test method for measurement of fracture toughness," in ASTM, Annual Book of Standards 3, 2011.

[19] “Markforged Metal 3D Printer: The Metal X 3D Printing System." [Online]. Available: https://markforged.com/3d-printers/metal-x. [Accessed: 08-Jun-2021].

[20] E. B. ROSENFELD, Azriel et TROY, "Visual texture analysis," Maryl. Univ., Coll. Park (USA). Comput. Sci. Cent., 1970.

[21] H. C. Lin, L. L. Wang, and S. N. Yang, "Extracting periodicity of a regular texture based 
on autocorrelation functions," Pattern Recognit. Lett., vol. 18, no. 5, pp. 433-443, 1997.

[22] K. Triconnet, K. Derrien, F. Hild, and D. Baptiste, "Parameter choice for optimized digital image correlation," Opt. Lasers Eng., vol. 47, no. 6, pp. 728-737, 2009.

[23] H. Sutton, M. A.; Orteu, J. J.; Schreier, Image correlation for shape, motion and deformation measurements: basic concepts, theory and applications. 2009.

[24] G. Besnard, F. Hild, and S. Roux, “Finite-element' displacement fields analysis from digital images: Application to Portevin-Le Châtelier bands," Exp. Mech., vol. 46, no. 6, pp. 789-803, 2006.

[25] Y. Sun, J. H. L. Pang, C. K. Wong, and F. Su, "Finite element formulation for a digital image correlation method," Appl. Opt., vol. 44, no. 34, pp. 7357-7363, 2005.

[26] F. Hild and S. Roux, "Comparison of Local and Global Approaches to Digital Image Correlation," Exp. Mech., vol. 52, no. 9, pp. 1503-1519, 2012.

[27] D. Claire, F. Hild, and S. Roux, "A finite element formulation to identify damage fields: The equilibrium gap method," Int. J. Numer. Methods Eng., vol. 61, no. 2, pp. 189-208, 2004.

[28] Z. Tomičević, F. Hild, and S. Roux, "Mechanics-aided digital image correlation," J. Strain Anal. Eng. Des., vol. 48, no. 5, pp. 330-343, 2013.

[29] A. Mendoza, J. Neggers, F. Hild, and S. Roux, "Complete mechanical regularization applied to digital image and volume correlation," Comput. Methods Appl. Mech. Eng., vol. 355 , pp. $27-43,2019$.

[30] F. dell'Isola et al., "Pantographic metamaterials: an example of mathematically driven design and of its technological challenges," Contin. Mech. Thermodyn., vol. 31, no. 4, pp. 851-884, Jul. 2019.

[31] J. Réthoré, S. Roux, and F. Hild, "An extended and integrated digital image correlation technique applied to the analysis of fractured samples: The equilibrium gap method as a mechanical filter," Eur. J. Comput. Mech., vol. 18, no. 3-4, pp. 285-306, 2009.

[32] M. D. C. Ferreira, W. S. Venturini, and F. Hild, "On the analysis of notched concrete beams: From measurement with digital image correlation to identification with boundary element method of a cohesive model," Eng. Fract. Mech., vol. 78, no. 1, pp. 71-84, 2011. 
[33] R. Vargas, X. Pinelli, B. Smaniotto, F. Hild, and R. B. Canto, "On the effect of sintering temperature on the fracture energy of an Alumina-Mullite-Zirconia castable at $600{ }^{\circ} \mathrm{C}$," J. Eur. Ceram. Soc., Feb. 2021.

[34] H. F. Leclerc H., Neggers J., Mathieu F., Roux S., “Correli 3.0.” 2015.

[35] M. Bornert et al., "Assessment of digital image correlation measurement errors: Methodology and results," Exp. Mech., vol. 49, no. 3, pp. 353-370, Dec. 2009.

[36] P. Doumalin, M. Bornert, and J. Crépin, "Characterisation of the strain distribution in heterogeneous materials," Mécanique Ind., vol. 4, no. 6, pp. 607-617, 2003.

[37] P. D. Nezhadfar, R. Shrestha, N. Phan, and N. Shamsaei, "Fatigue behavior of additively manufactured 17-4 PH stainless steel: Synergistic effects of surface roughness and heat treatment," Int. J. Fatigue, vol. 124, no. February, pp. 188-204, 2019.

[38] H. Mirzadeh and A. Najafizadeh, "Hot deformation and dynamic recrystallization of 174 PH stainless steel," ISIJ Int., vol. 53, no. 4, pp. 680-689, 2013.

[39] H. Mirzadeh, A. Najafizadeh, and M. Moazeny, "Flow curve analysis of 17-4 PH stainless steel under hot compression test," in Metallurgical and Materials Transactions A: Physical Metallurgy and Materials Science, 2009, vol. 40, no. 12, pp. 2950-2958. 


\section{Appendix A: Evaluation of mean strain rates}

Figure A1 shows the temporal changes of the mean longitudinal strain computed over the ROIs of the five analyzed tests (Figure7). The mean strain rate was computed in the linear regime (i.e., prior to crack initiation that induced accelerated strain variations). In the present analyses, longitudinal strain rates ranging from $1.2 \times 10^{-5}$ to $10-4 \mathrm{~s}^{-1}$ were investigated (Table A1). In that range, Portevin Le Chatelier effects were observed.

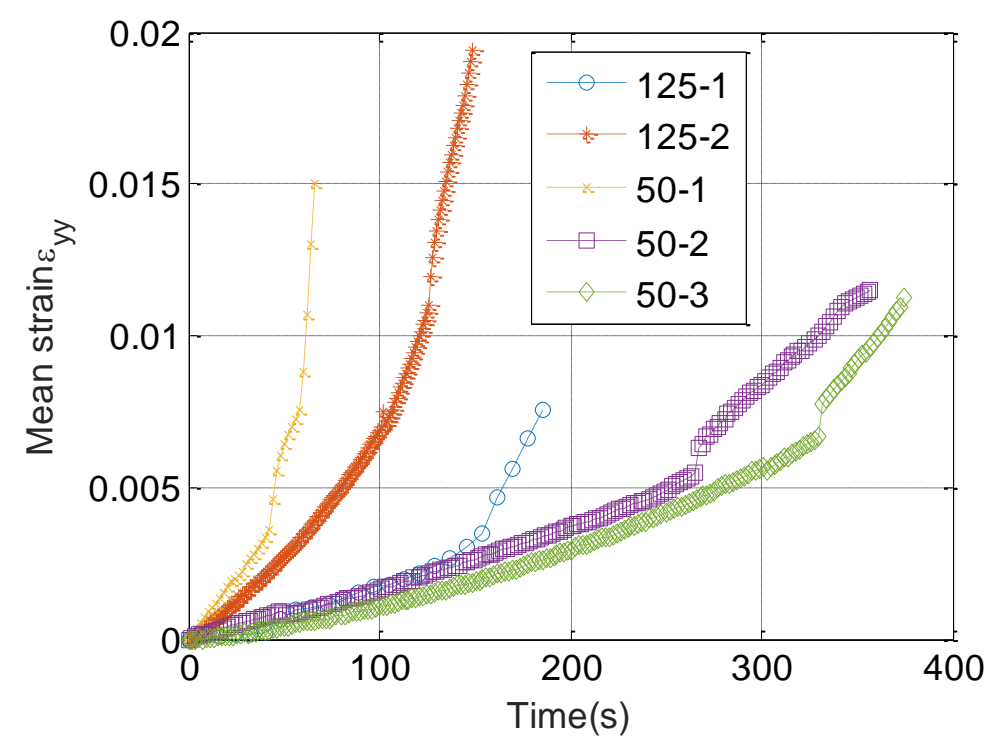

Figure 1. Longitudinal mean strain history for the five tests.

Table 1. Average longitudinal strain rates computed from Figure A1.

\begin{tabular}{|c|c|}
\hline Specimen designation & $\dot{\boldsymbol{\varepsilon}}\left(\mathbf{s}^{-\mathbf{1}}\right)$ \\
\hline $\mathbf{1 2 5}-1$ & $1.2 \times 10^{-5}$ \\
\hline $\mathbf{1 2 5}-2$ & $5.8 \times 10^{-5}$ \\
\hline $\mathbf{5 0 - 1}$ & $10^{-4}$ \\
\hline $\mathbf{5 0 - 2}$ & $1.2 \times 10^{-5}$ \\
\hline $\mathbf{5 0 - 3}$ & $1.4 \times 10^{-5}$ \\
\hline
\end{tabular}

Contrary to the critical NODs that were found to be very close for all five samples (Table 5), Figure 7 shows that for the samples with four wall contours (i.e., 125-1, 125-2 and 50-1), the mean strains to failure varied a lot. Conversely, the mean strains to failure for the two samples with two wall contours (i.e., 50-2 and 50-3) were very close. 